\title{
Spherically Symmetric Cosmology in Presence of Perfect Fluid
}

\author{
Dr.Avinash Nagpure ${ }^{1}$,Manjushree Muley $^{2}$, , Dr.S.D.Tade ${ }^{3}$ \\ ${ }^{2}$ Department of Mathematics, Research Scholar Rashtrasant Tukadoji Maharaj NagpurUniversity, Nagpur \\ ${ }^{1,3}$ Department of Mathematics, Jawaharlal Nehru Arts, Commerce \& Science College. Wadi, Nagpur-440023(India)
}

DOI: 10.46335/IJIES.2020.5.8.6

\begin{abstract}
The Spherically Symmetric Model has been obtained in the general theory of relativity. The source for energy-momentum tensor is assumed a perfect fluid. The field equation has been solved by using a special form of the average scale factor $R(t)=\left[\left(t-t_{0}\right)^{2}+\frac{t_{0}}{1-\beta}\right]^{\frac{1}{1-\beta}}$ proposed by Cai et
\end{abstract} al. The physical properties and the bouncing behaviour of the model are also discussed.

Keywords- Spherically symmetric space time, Bouncing Universe.

\section{I- INTRODUCTION}

In this paper bouncing behaviour of Spherically Symmetric cosmological model has been obtained in the general theory of relativity. This work is organised as follows in Section 2. The metric and field equations have been presented. The field equations have been solved in section 3 by using a physical condition that the expansion scalar $\theta$ is proportional to shear scalar $\sigma$ and the special form of average scalar factor $R(t)=\left[\left(t-t_{0}\right)^{2}+\frac{t_{0}}{1-\beta}\right]^{\frac{1}{1-\beta}}$ Proposed by Cai et. al. (2011). The physical and geometrical behaviour of the model have been discussed in section 4 in the last section 5 concluding remarks have been expressed.

\section{II- METRIC AND FIELD EQUATIONS}

We consider five dimensional spherically symmetric metric of the form

$d s^{2}=d t^{2}-a_{1}^{2} d r^{2}-a_{2}^{2}\left[d \theta_{1}^{2}+\sin ^{2} \theta_{1} d \theta_{2}^{2}+\sin ^{2} \theta_{1} \sin ^{2} \theta_{2} d \theta_{3}^{2}\right]$

Where the metric functions $a_{1}, a_{2}$ are functions of cosmic time ' $\mathrm{t}$ ' only.

The energy - momentum tensor for a perfect fluid is

$$
T_{i}^{j}=(\rho+p) u_{i} u^{j}-p g_{i}^{j}
$$

where $\mathrm{p}$ is the pressure, $\rho$ is the energy density and $g_{i}{ }^{j}$ is a metric tensor. In co-moving coordinate system, $u^{i}$ are the four co-moving velocity vectors which satisfy the condition

$$
u_{i} u^{i}=0, \quad \text { for } \quad i=1,2,3,4
$$

and

$$
u_{i} u^{i}=1, \text { for } i=0
$$

From equation (2) the components of energy momentum tensor are 


$$
T_{0}^{0}=\rho, T_{1}^{1}=T_{2}^{2}=T_{3}^{3}=T_{4}^{4}=-p
$$

With the help of equation (3) the energy momentum tensor takes the form,

$$
T_{i}^{j}=\operatorname{diag}(\rho,-p,-p,-p,-p)
$$

For the perfect fluid, $\mathrm{p}$ and $\rho$ are related by equation of state

$p=\omega \rho, \quad 0 \leq \omega \leq 1$

The Einstein's field equations are given by

$R_{i}^{j}-\frac{1}{2} g_{i}^{j} R=-T_{i}^{j}$

where $R_{i}{ }^{j}$ is a Ricci tensor, $\mathrm{R}$ is the Ricci scalar.

The Ricci scalar for the spherically symmetric metric is given by

$R=2\left(\frac{\ddot{a}_{1}}{a_{1}}+\frac{3 \dot{a}_{1} \dot{a}_{2}}{a_{1} a_{2}}+3 \frac{\ddot{a}_{2}}{a_{2}}+3\left(\frac{\dot{a}_{2}}{a_{2}}\right)^{2}+\frac{3}{a_{2}^{2}}\right)$

With the help of equations (4) and (5) the field equations (6) for the metric (1) are

$$
\begin{aligned}
& \frac{3 \dot{a}_{1} \dot{a}_{2}}{a_{1} a_{2}}+3\left(\frac{\dot{a}_{2}}{a_{2}}\right)^{2}+\frac{3}{a_{2}{ }^{2}}=\rho \\
& 3 \frac{\ddot{a}_{2}}{a_{2}}+3\left(\frac{\dot{a}_{2}}{a_{2}}\right)^{2}+\frac{3}{a_{2}{ }^{2}}=-\omega \rho
\end{aligned}
$$

$$
\frac{\ddot{a}_{1}}{a_{1}}+2 \frac{\ddot{a}_{2}}{a_{2}}+\frac{2 \dot{a}_{1} \dot{a}_{2}}{a_{1} a_{2}}+\left(\frac{\dot{a}_{2}}{a_{2}}\right)^{2}+\frac{1}{a_{2}^{2}}=-\omega \rho
$$

Here the over dot represents the differentiation with respect to $t$.

\section{III- SOLUTIONS OF FIELD EQUATIONS}

The field equations (7) to (9) are a system of three highly non-linear differential equations in four unknown's $a_{1}, a_{2}, \rho, \omega$.The system is thus initially undetermined. So, to obtain the determinate solution to above field equations we need one extra physical condition to solve the field equations completely. So, hence we assume the condition expansion scalar $(\theta)$ is proportional to the shear scalar $(\sigma)$. This condition may leads to (Chakraborty S. and Ckakraborty A.K. 1992)

where $\eta, \mathrm{m}$ are constants

$$
a_{1}=\eta\left(a_{2}\right)^{m}
$$

Here for simplicity and without loss of generality, we assume that

$$
\eta=1
$$

Hence, we have

$$
a_{1}=\left(a_{2}\right)^{m},(m \neq 1)
$$

Collins et al. have pointed out that for spatially homogenous metric, the normal congruence to the homogenous expansion satisfies that the condition $\frac{\sigma}{\theta}$ is constant (Collins, Giass et al. 1980).

In cosmology, the constant deceleration parameter is commonly used by several researchers (Akarsu and Kilinc 2010, Akarsu and Kilinc 2010, Saha, Amirhashchi et al. 2012, Kumar and Singh 2011), as it duly gives a power law for metric function or corresponding quantity.

The motivation to choose time-dependent deceleration parameter (Deceleration parameter) is the fact that the expansion of the universe was decelerating in the past and accelerating at present as observed by recent observations of Type Ia Supernova (Riess, Filippenko et al. 1998, Perlmutter, Aldering et al. 1999, Tonry, Schmidt et al. 2003) and CMB anisotropies (Bennett, Halpern et al. 2003, Hanany, Ade et al. 2000). Also, the transition red shift from deceleration expansion to accelerated expansion is about 0.5 . Now for a Universe which was decelerating in the past and accelerating at present, the Deceleration parameter must show signature flipping (Amendola 2003, Padmanabhan and Choudhury 2003, Riess, Nugent et al. 2001,). So, in general, the Deceleration parameter is not a constant but time variable. The motivation to choose the following scale factor is that it provides a time-dependent Deceleration parameter. So, we use a special form of deceleration parameter as 
$q=-\frac{R \ddot{R}}{R^{2}}=-1+\frac{d}{d t}\left(\frac{1}{H}\right)=-1+\frac{1}{2}\left[(1-\beta)-\frac{t_{0}}{\left(t-t_{0}\right)^{2}}\right], \beta<1$

where $\mathrm{R}$ is average scale factor of the universe .

This form is proposed by Cai et al. (Cai, Qiu et al. 2007) and then modified by Sadatian (Sadatian 2014).

Integrating twice equation (11), the average scale factor which is time dependent is

$R(t)=\left[\left(t-t_{0}\right)^{2}+\frac{t_{0}}{1-\beta}\right]^{\frac{1}{1-\beta}}$

where $\mathrm{t}_{0}$ is initial time and $\beta<1$ is constant.

For the metric (1), the scale factor $\mathrm{R}$ is given by

$$
R(t)=\left(a_{1} a_{2}^{3}\right)^{\frac{1}{4}}
$$

Now, from the equations (12) and (13), we obtain $\left(a_{1} a_{2}^{3}\right)^{\frac{1}{4}}=\left[\left(t-t_{0}\right)^{2}+\frac{t_{0}}{1-\beta}\right]^{\frac{1}{1-\beta}}$

$a_{1} a_{2}^{3}=\left[\left(t-t_{0}\right)^{2}+\frac{t_{0}}{1-\beta}\right]^{\frac{4}{1-\beta}}$

In view of equation (10), it becomes

$$
\begin{aligned}
& a_{2}^{m} a_{2}^{3}=\left[\left(t-t_{0}\right)^{2}+\frac{t_{0}}{1-\beta}\right]^{\frac{4}{1-\beta}} \\
& a_{2}=\left[\left(t-t_{0}\right)^{2}+\frac{t_{0}}{1-\beta}\right]^{\frac{4}{(1-\beta)(m+3)}}
\end{aligned}
$$

Using equation (14), equation (10) leads to

$$
a_{1}=\left[\left(t-t_{0}\right)^{2}+\frac{t_{0}}{1-\beta}\right]^{\frac{4 m}{(1-\beta)(m+3)}} \text {. }
$$

With the help of equations (14) and (15), the metric (1) becomes

$d s^{2}=d t^{2}-\left[\left(t-t_{0}\right)^{2}+\frac{t_{0}}{1-\beta}\right]^{\frac{8 m}{(1-\beta)(n+3)}} d r^{2}-\left[\left(t-t_{0}\right)^{2}+\frac{t_{0}}{1-\beta}\right]^{\frac{8}{(1-\beta)((n+3)}}\left[d \theta_{1}^{2}+\sin ^{2} \theta_{1} d \theta_{2}^{2}+\sin ^{2} \theta_{1} \sin ^{2} \theta_{2} d \theta_{3}^{2}\right]$

which represents spherically symmetric 5-dimensional model in general relativity.

\section{IV- PHYSICAL PROPERTIES OF THE MODEL}

For the spherically symmetric model (16), the physical quantities such as spatial volume V, Hubble parameter $\mathrm{H}$, expansion scalar $\theta$, mean anisotropy $A_{m}$, shear scalar $\sigma^{2}$, energy density $\rho$, and the equation of state parameter $\omega$ are obtained as follows:

The average scale factor $\mathrm{R}$ and volume scalar $\mathrm{V}$ are given by

$$
R^{4}=V=a_{1} a_{2}^{3}
$$

The generalized Hubble's parameter $\mathrm{H}$ is defined by

$$
H=\frac{\dot{R}}{R}=\frac{1}{4}\left(H_{0}+3 H_{1}\right)
$$

where $H_{0}=\frac{\dot{a}_{1}}{a_{1}}$ and $H_{1}=\frac{\dot{a}_{2}}{a_{2}}$ are the directional Hubble's parameter. An overhead dot denotes the differentiation with respect to cosmic time $t$.

The expansion scalar $\theta$ and Shear scalar $\sigma$ are given by

$$
\theta=u^{i} ;_{i}=\left(\frac{\dot{a}_{1}}{a_{1}}+3 \frac{\dot{a}_{2}}{a_{2}}\right)=4 H
$$


and

$\sigma^{2}=\frac{1}{2}\left[\sum_{i=1}^{n} H_{i}^{2}-3 H^{2}\right]$.

The spatial volume is in the form

$$
V=R^{4}=\left[\left(t-t_{0}\right)^{2}+\frac{t_{0}}{1-\beta}\right]^{\frac{4}{1-\beta}}
$$

The Hubble parameter is

$$
H=\frac{2\left(t-t_{0}\right)}{1-\beta}\left[\left(t-t_{0}\right)^{2}+\frac{t_{0}}{1-\beta}\right]^{-1}
$$

From fig 4.1 (a), the Hubble parameter $\mathrm{H}<0$, for $\mathrm{t}<1$ and $\mathrm{H}>0$, for $\mathrm{t}>1$ indicating that $\mathrm{H}$ passes across zero $(\mathrm{H}=0)$ at $\mathrm{t}=1$, which represents that the universe is bouncing at $\mathrm{t}=1$.

The expansion scalar is

$$
\theta=\frac{8\left(t-t_{0}\right)}{1-\beta}\left[\left(t-t_{0}\right)^{2}+\frac{t_{0}}{1-\beta}\right]^{-1}
$$

The mean anisotropy parameter is

$$
A_{m}=\frac{3(m-1)^{2}}{(m+3)^{2}}=\text { const } \neq 0, \text { for } m \neq 1
$$

The shear scalar is

$$
\sigma^{2}=\frac{16(m-1)^{2}\left(t-t_{0}\right)^{2}}{(1-\beta)^{2}(m+3)^{2}}\left[\left(t-t_{0}\right)^{2}+\frac{t_{0}}{1-\beta}\right]^{-2}
$$

It is observed that $\lim _{t \rightarrow \infty} \frac{\sigma^{2}}{\theta^{2}}=\frac{(m-1)^{2}}{4(m+3)^{2}} \neq 0$ for $m \neq 1$
The mean anisotropy parameter $A_{m}$ is constant and $\lim _{t \rightarrow \infty} \frac{\sigma^{2}}{\theta^{2}} \neq 0$ is also constant. Hence the model is anisotropic throughout the evolution of the universe except at $\mathrm{m}=1$ i.e., the model does not approach isotropy.

The matter- energy density is given by

$\rho=\frac{192(m+1)\left(t-t_{0}\right)^{2}}{(1-\beta)^{2}(m+3)^{2}}\left[\left(t-t_{0}\right)^{2}+\frac{t_{0}}{1-\beta}\right]^{-2}+3\left[\left(t-t_{0}\right)^{2}+\frac{t_{0}}{1-\beta}\right]^{-\frac{-8}{(1-\beta)(m+3)}}$.

The equation of state parameter (EoS) is given by

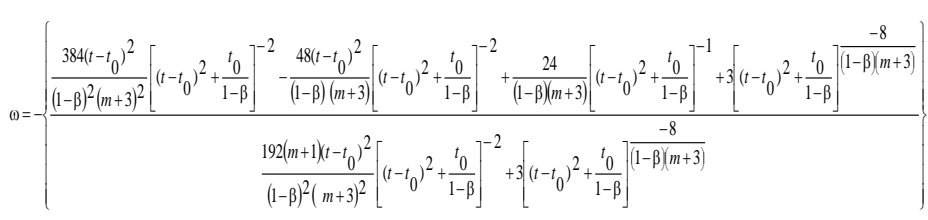

To study the physical properties of spherically symmetric cosmological model, plots of time versus (a) average scale factor (b) spatial volume (c) Hubble parameter (d) energy density (e)EoS parameter for the values are shown in Fig (4.1)

(a)

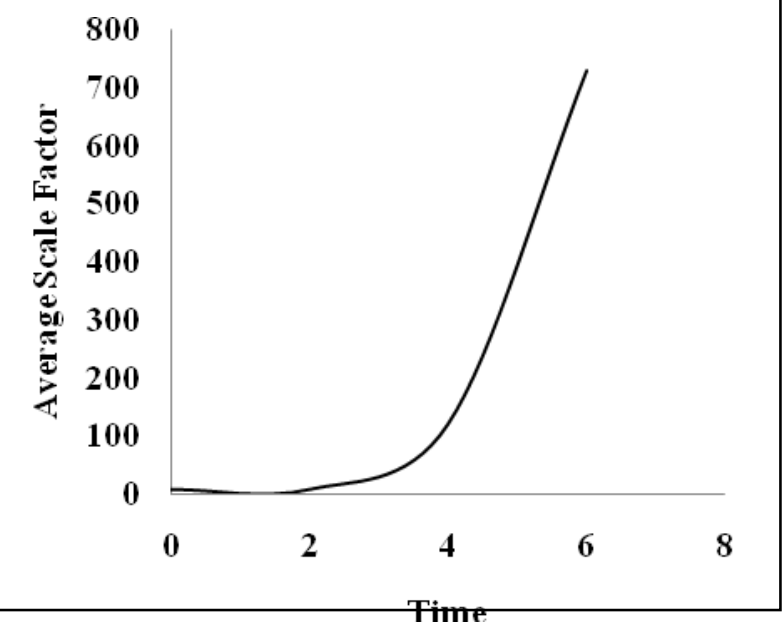



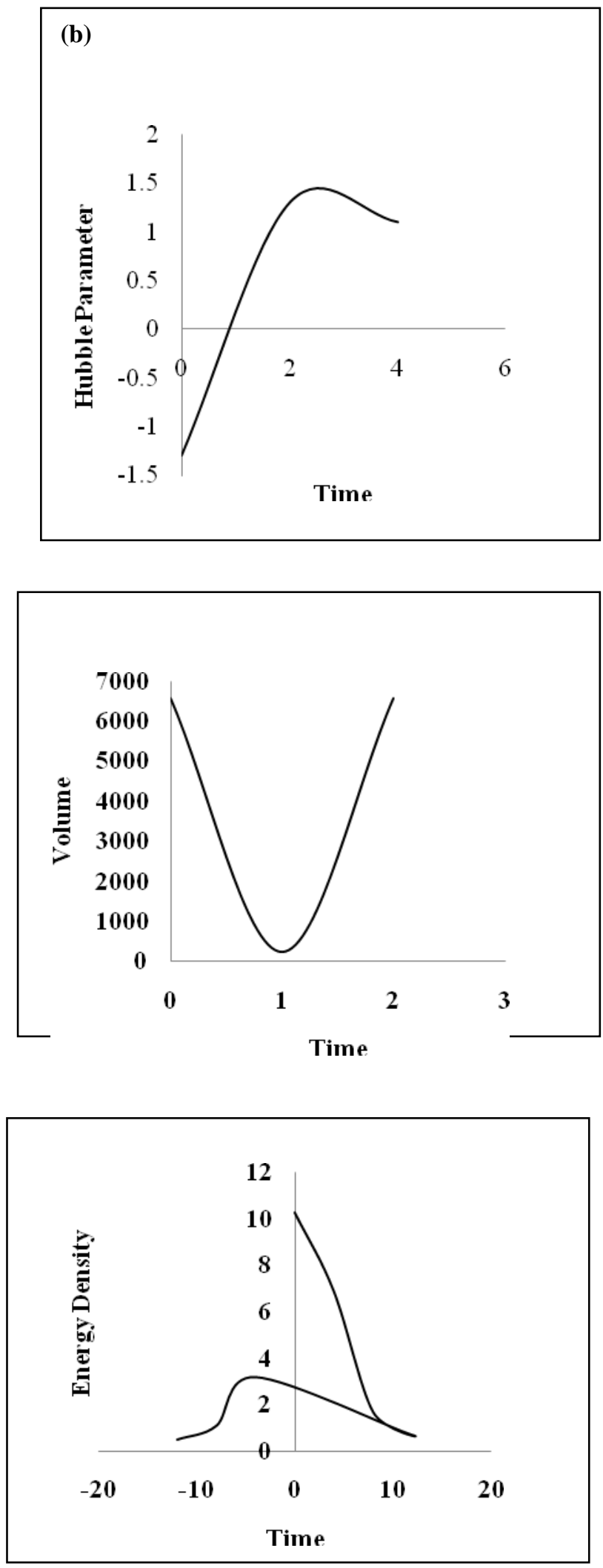

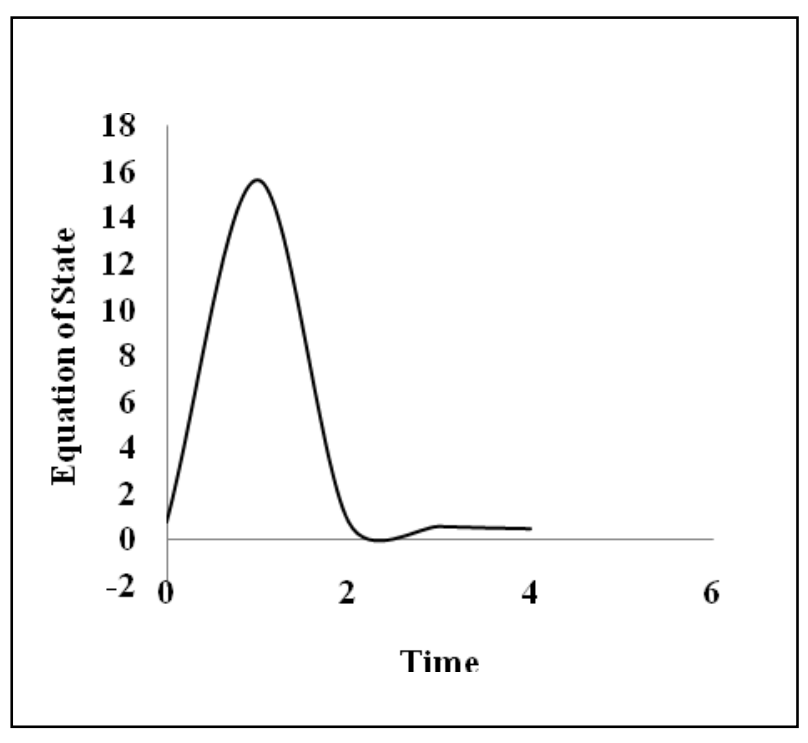

Fig 4.1 Plots of time versus - (a) Average Scale factor (b) Spatial Volume (c) Hubble Parameter (d) Energy Density (e) EoS Parameter for the values $\beta=0.5, t_{0}=1, m=2$.

From Fig 4.1 (a), in the earlier stage, the average scale factor $(\mathrm{R})$ is strictly decreasing $(\mathrm{R}(\mathrm{t})<0)$ and in the expanding phase, it increases rapidly $(R(t)>0)$. Hence our model is bouncing at some finite time $t=1(\mathrm{R}(\mathrm{t})=$ 0)

From Fig 4.1 (d) the energy density decreases at the early stage of evolution when $t<1$ and goes into the hot Big Bang era. The model bounces at $t=1$ and after bouncing the energy density rapidly increases for $t>1$.

It is seen that from fig.4.1 (e), before bouncing (at point $\mathrm{t}=1)$, the EoS parameter $\omega<-1$ and after the bounce, $\omega>-1$ for $t>1$. The equation of state parameter of the universe crosses from $\omega<-1$ to $\omega>-1$. Hence, our model is bouncing at $t=1$. Thus, it is observed that, a bouncing universe model has an initial narrow state by a non - zero minimal radius and then develops to an expanding phase. After the bounce, the universe enters into the hot Big - Bang era.

\section{V- CONCLUSIONS}

The Spherically symmetric cosmological model has been investigated in the general theory of relativity. The source for energy - momentum tensor is a perfect fluid. The field equations have been solved by using time dependent deceleration parameter. The mean anisotropy 
parameter $\mathrm{A}_{\mathrm{m}}$ is constant and $\lim _{t \rightarrow \infty} \frac{\sigma^{2}}{\theta^{2}} \neq 0$ is constant, hence the model is anisotropic throughout the evolution of the universe except at $m=1$. It is interesting to note that a bouncing universe model has an initial narrow state by non -zero minimal radius and then develops to expanding phase. After the bounce, the universe enters into the Hot Big-Bang era. The model has a bounce at some finite time $\mathrm{t}=\mathrm{t}_{0}$. In particular, for the values $\beta=0.5, t_{0}=1, m=2$ the model is bouncing at finite time $\mathrm{t}_{0}=1$.

\section{REFERENCES}

[1] Riess, A.G.;Filippenko, A. V.;Challis,P.;Clocchiatti, A.; Diercks, A.;Garnavich,P.M.;Gilliland, R.L.; Hogan, C.J.; Jha, S.; Kirshner,R.P.; et al . (1998). "Observational evidence from supernovae for an accelerating universe and a cosmological constant", Astronom. J., 116, 1009-1038.

[2] Perlmutter, S;Aldering, G.; Goldhaber, G; Knop, R.A.;Nugent, P.;Castro, P.G.; Deustua, S.; Fabbro, S.; Goobar, A.; Groom, D.E. et al. (1999 ). "Measurementof from 42 high-redshift supernovae", Astrophys.J. 517, No.2, 565-586.

[3] Peebles, P. J. E.; Ratra, B, (2003). "The cosmological constant and dark energy ", Rev. Mod. Phys., 75, 559606.

[4] Sahni, V.; Starobinsky, A.A. (2000). "The case for a positive cosmological Lambda-term”, Int.J. Mod Phys. D, 9, 373-443.

[5] Li, M.; Li, X.; Wang, Y. (2011). "Dark energy", Commun. Theor. Phys., 56, 525-604.

[6] Kowalski, M.; Rubin, D.; Aidering, G.; Agostinho, R.J.; Amadon, A.; Amanullah, R.; Balland, C.; Barbary, K; Blanc, G.; Challis, P.J.; et al. (2008). "Improved cosmological constants from new, old, and combined supernova data sets ", Astrophys.J., 686, 749-778.

[7] Nojiri, S.; Odintsov,S.D.; Tsujikawa,S.; (2005). "Propertiesof singularities in the (phantom) dark energy universe”, Phys. Rev. D, 71, 063004, Universe 2015, 135.

[8] Bamba, K.; Capozziello, S.; Nojiri, S.;,( 2012), “ Obinesov, S.; D. ', "Dark energy cosmology :The equivalent description via different theortical models and cosmography test”, Astrophys. Sci., 342, 155-228.
[9] Nojiri, S.; Odintsov, S.D.(2011). "Unified cosmic history in modified gravity: Fromf $(R)$ theory to Lorentz non invariant models". Phys. Rep., 505, 59-144.

[10] Capozziello, S.; de Laurentis, M. (2011). "Extended theories of gravity”. Phys. Rep., 509, 167-320.

[11]Nojiri, S.; Odintsov, S. D. (2007). "Introduction to modified gravity and gravitational alternative for dark energy”. Int. J. Geom. Meth. Mod. Phys., 4, 115-145.

[12] Sadatian S. D. (2014). "Rip singularity scenario and bouncing universe in a Chaplygin gas dark energy model", Int. J. Theo. Phys., 53, 675-684. DOI 10. 1007/s 10773-013-1855-1.

[13] Bamba K., Makarenko A. N. (2013). "Bounce cosmology from $f(R)$ gravity and $f(R)$ bi-gravity”, arXiv 1309.3748v2[hep-th].

[14] Astashenok A. V. (2014). "Effective dark energy models and dark energy models with bounce in frames of $F(T)$ gravity", Astrophys. Spa. Sci. 351,377-383. DOI: 10. 1007/s 10509-014-1846-6.

[15] Solomans d., Dunsby P. K. S., Ellis G. F. R. (2006). "Bounce behaviour in Kantowski-Sachs and Bianchi cosmologies", Classical and Quan. Grav. 23, (23); arXiv: [gr-qc] 0103087v2,: DOI 10.1088/0264-9381/23/23/001.

[16] Silva G. F.,Piattella O. F. , Fabris J. C., Casarini L., Barbosa T. O. (2014). "Bouncing solutions in Rastalls theory with a barotropic fluid”, Grav. Cosm. 19, 156162, 2013. arXiv.1212.6954v3 [gr-qc]. 10. $1142 / S 02773231400783$.

[17]Brevik I., Obukhov V. V., Timoshkin A. V. (2014). "Bounce universe induced by an inhomogeneous dark fluid coupled with dark matter", Mod. Phys. Lett. A 29, Issue 15, 1450078. DOI: (2015) Universe 1, 24-37,; arXiv $1404.11887 v 1$ [gr-qc].

[18] Singh T., Chaubuy R., Singh A. ( 2015). "k-essence cosmologies in Kantowski - Sach and Bianchi spacetimes", Canadian J. Of Phys., 93, (11) 1319-1323. DOI: 10.1139/cjp-2015-0001.

[19] S.Chakraborty and A.K. Chakraborty: (1992). J. Phys.33, 2336.

[20] Collins C. B., Glass E. N., Wilkinson D. A., " Exact spatially homogenous cosmologies”, Gen. Relat. Grav. Vol. 12, No. 10, pp. 805-823, 1980.

[21] Akarsu, O., Kilinc, C. B. (2010). "LRS Bianchi type - I models with anisotropic dark energy and constant deceleration parameter", Gen. Relat. Gravit. 42, 119, a. 
[22] Akarsu, O., Kilinc , C. B. (2010). "Bianchi type - III models with anisotropic dark energy", General Relativity and Gravitation, Vol. 42, No. 4, pp. 763-775. Doi: 10.1007/s10714-009-0878-7.

[23] Saha, B., Amirhashchi, H., Pradhan, A. (2012). "Twofluid scenario for dark energy models in an FRW universe-revisited", Astrophys. Space Sci., Vol. 342, pp. 257- 267.

[24] Kumar's., Singh, C.P. (2011). “Anisotropic dark energy models with constant deceleration parameter”, Gen. Rel. Grav., 43, 1427,.

[25] Tonry, J. L., Schmidt, B.P., Barris, B., et al. (2003). (Supernova Search Team Collaboration), "Cosmological results from high $-z$ supernovae”, Astrophys. J., 594, No. I, 1-24.

[26] Bennett, C. L., Halpern, M., Hinshaw, G., et al. (2003). "First year Wilkinson microwave anisotropy probe (WMAP) Observations: Preliminary maps and basic results", Astrophys. J. Suppl., 148, 1.

[27] Hanany, S., et al., "A measurement of the cosmic microwave background anisotropy on angular scales of 10 arc minutes to 5 degrees ", Astro-ph/0005123.

[28] Amendola, L. (2003). "Acceleration at $z>I$ ?" Monthly notices of the Royal Astronomical Society, 342, 221-226

[29] Padmanabhan, T., andRoychowdhury, t. (2003). "A theoreticians analysis of the supernova data and the limitations in determining the nature of dark energy", Monthly Notices of the Royal Astronomical Society, 344, 823-834.

[30] Riess, A. G., Nugent, P.E., Gilliland, R.L. et al. (2001). "The Farthest known supernova: Support for an accelerating universe and a glimpse of the epoch of deceleration", The Astrophysical Journal, Vol. 560, No, 1, pp. 49. 